\title{
Effect of an aqueous extract of Ajuga iva on glycaemia, reverse cholesterol transport and atherogenic ratios in rats with streptozotocin-induced diabetes
}

\author{
D. Taleb-Senouci ${ }^{1}$, D. Krouf ${ }^{1}$, H. Ghomari ${ }^{1}$, M. A. Lacaille-Dubois ${ }^{2}$ and M. Bouchenak ${ }^{1}$ \\ ${ }^{1}$ Laboratoire de Nutrition Clinique et Métabolique, Faculté des Sciences, Université Es Sénia, Oran, Algeria and \\ ${ }^{2}$ Laboratoire de Pharmacognosie, Faculté de Pharmacie, Université de Bourgogne, Dijon, France
}

Experimental evidence suggests that hyperglycaemia is commonly associated with hyperlipidaemia ${ }^{(1,2)}$. The present study was undertaken to investigate the effect of an aqueous extract of Ajuga iva L. Schreiber (Lamiaceae; Ai) on blood glucose, serum and lipoprotein lipid profiles and lecithin:cholesterol acyltransferase (LCAT) activity in rats with streptozotocin-induced diabetes.

Twelve rats with diabetes were divided into two groups that were fed a casein diet either with or without an $A i$ supplement (5 g/kg diet) for 4 weeks. Experimental diabetes was induced by intraperitoneal injection of streptozotocin as a single dose of $60 \mathrm{mg} / \mathrm{kg}$ body weight. HDL subfractions were separated by differential dextran sulphate- $\mathrm{MgCl}_{2}$ precipitation and LCAT activity was determined by conversion of $\left[{ }^{3} \mathrm{H}\right]$ cholesterol (unesterified; UC) to $\left[{ }^{3} \mathrm{H}\right]$ cholesteryl esters $(\mathrm{CE})$.

$A i$ treatment significantly decreased glycaemia (-41\%) and liver total cholesterol (TC; $-33 \%)$, TAG (-30\%) and phospholipids (PL; $-47 \%)$. In the $A i$-treated rats compared with the untreated rats hypocholesterolaemia $(-33 \%)$ and hypotriacylglycerolaemia $(-72 \%)$

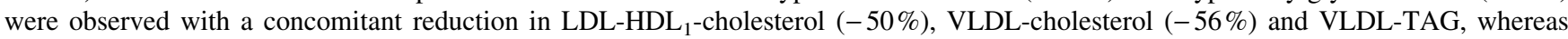
HDL-cholesterol remained unchanged for both groups. Moreover, plasma apoB concentration was 2-fold lower, while that of apoA was 2.4-fold higher.

\begin{tabular}{lcccr}
\hline Group.... & \multicolumn{2}{c}{ Untreated } & \multicolumn{2}{c}{$A i$-treated } \\
\cline { 2 - 5 } & Mean & $\mathrm{SE}$ & Mean & $1.86^{*}$ \\
\hline LCAT $(\mathrm{nmol} / \mathrm{ml}$ per $\mathrm{h})$ & 10.0 & 1.93 & $2.31^{* * *}$ & 1.29 \\
ApoA $(\mathrm{g} / \mathrm{l})$ & 0.98 & 0.12 & $0.27 * *$ & 0.24 \\
$\mathrm{HDL}_{3}$-PL $(\mathrm{mmol} / \mathrm{l})$ & 0.90 & 0.12 & $0.09 * *$ & 0.08 \\
$\mathrm{HDL}_{3}$-UC $(\mathrm{mmol} / \mathrm{l})$ & 0.21 & 0.02 & 0.56 & 0.03 \\
$\mathrm{HDL}_{2}$-CE $(\mathrm{mmol} / \mathrm{l})$ & 0.39 & 0.03 & 0.19 \\
\hline Mean values were significantly different from those for the untreated group: $* P<0.05, * * P<0.01, * * * P<0.001$.
\end{tabular}

LCAT activity was 1.5 -fold higher in the $A i$-treated rats than in the untreated rats. Moreover, $\mathrm{HDL}_{3}$ - $\mathrm{PL}$ and $\mathrm{HDL}_{3}$-UC were decreased by $57 \%$ and $70 \%$ respectively, whereas $\mathrm{HDL}_{2}-\mathrm{CE}$ was similar for both groups. Also, the atherogenic ratios TC: HDL-cholesterol, VLDLLDL-cholesterol : HDL-cholesterol and apoB:apoA were decreased by $31 \%, 46 \%$ and $79 \%$ respectively in $A i$ treated rats $v$. untreated rats.

These results suggest that $A i$ treatment is effective in decreasing the level of glycaemia and attenuating dyslipidaemia in rats with streptozotocin-induced diabetes by reducing plasma lipids and inversely increasing reverse cholesterol transport.

1. Annida B \& Stanely Mainzen Prince P (2004) J Med Food 7, 153-156.

2. Tunali S \& Yanardag R (2006) Pharmacol Res 53, 271-277. 